\title{
Central localization of plasticity involved in appetitive conditioning in Lymnaea
}

\author{
Volko A. Straub, ${ }^{1}$ Benjamin J. Styles, Julie S. Ireland, Michael O'Shea, and \\ Paul R. Benjamin \\ Sussex Centre for Neuroscience, Department of Biology and Environmental Science, School of Life Sciences, University of Sussex, \\ Falmer, Brighton, BN1 9QG, United Kingdom
}

\begin{abstract}
Learning to associate a conditioned (CS) and unconditioned stimulus (US) results in changes in the processing of CS information. Here, we address directly the question whether chemical appetitive conditioning of Lymnaea feeding behavior involves changes in the peripheral and/or central processing of the CS by using extracellular recording techniques to monitor neuronal activity at two stages of the sensory processing pathway. Our data show that appetitive conditioning does not affect significantly the overall CS response of afferent nerves connecting chemosensory structures in the lips and tentacles to the central nervous system (CNS). In contrast, neuronal output from the cerebral ganglia, which represent the first central processing stage for chemosensory information, is enhanced significantly in response to the CS after appetitive conditioning. This demonstrates that chemical appetitive conditioning in Lymnaea affects the central, but not the peripheral processing of chemosensory information. It also identifies the cerebral ganglia of Lymnaea as an important site for neuronal plasticity and forms the basis for detailed cellular studies of neuronal plasticity.
\end{abstract}

An important aim in studies of learning and memory is the identification of sites in the nervous system in which learninginduced changes occur. In vertebrate models of learning and memory, a number of loci for synaptic and cellular plasticity have been identified (see Rosenzweig 1996; Christian and Thompson 2003). This type of organization may also occur in some types of invertebrate model systems in which more complex behavioral networks have been investigated. For instance, in the feeding systems of gastropod mollusks, changes in neuronal activity and cellular properties that were recorded following tactile conditioning occur at all levels of the system, including central sensory pathways, modulatory interneurons, central pattern generator (CPG) interneurons, and motoneurons (see Kemenes et al. 1997; Staras et al. 1999; Jones et al. 2003; Mozzachiodi et al. 2003) involving several possible sites of plasticity.

As part of a study aimed at locating the cellular mechanisms of associative memory formation in the feeding system of the pond snail Lymnaea, we have examined the neural pathways that mediate the responses to the chemical conditioned (CS; amyl acetate [AA]) and unconditioned stimulus (US; sucrose) cues used for one trial associative conditioning. By recording these responses on peripheral sensory nerves and on selected central connectives, we have investigated both the basic responsiveness of the peripheral and central pathways involved in activating feeding in naive animals and identified the sites of change underlying the enhanced behavioral response to the CS after conditioning (Alexander Jr. et al. 1984; Kemenes et al. 2002).

Previous evidence suggests that neuronal changes underlying behavioral plasticity of feeding occur somewhere on the pathway between the sensory periphery and the output of the cerebral ganglia (Benjamin et al. 2000). There are two processing stages in this pathway that are potentially involved in chemical conditioning. The first is located in the peripheral nervous sys-

\footnotetext{
${ }^{1}$ Corresponding author.

E-mail v.straub@sussex.ac.uk; fax 44-1273-678535.

Article published online ahead of print. Article and publication date are at http://www.learnmem.org/cgi/doi/10.1101/lm.77004.
}

tem where the initial sensing of the CS and US by chemoreceptors in the lips and tentacles occurs (Zaitseva and Bocharova 1981; Nakamura et al. 1999). The second is in the cerebral ganglia, the first major central processing center for the chemosensory information arriving from the periphery, and also the location of modulatory interneurones important for the activation and modulation of feeding (see McCrohan 1984; Yeoman et al. 1994a,b; Kemenes et al. 2001). Both these potential sites of plasticity were tested in the present experiments.

We show that a single training trial leading to appetitive conditioning of feeding in Lymnaea does not alter the response to the CS in the periphery but causes significant changes in the central processing of chemosensory information within the cerebral ganglia. These ganglia appear to contain a sensory "gating" mechanism that in control animals prevents information about the CS reaching the feeding CPG. This block of CS sensory information to the feeding CPG is removed after conditioning.

\section{Results}

\section{Conditioning does not alter peripheral processing of chemosensory information}

Chemosensory information from the sensory epithelia of the lips and tentacles enters the central nervous system (CNS) at the level of the cerebral ganglia via the median and superior lip nerves and the tentacle nerve. The feeding CPG is contained in the buccal ganglia and the link between the cerebral ganglia and the feeding CPG is provided by the cerebral-buccal connective (cbc). To enable the CS to affect the feeding behavior, training must modify the chemosensory responses to the CS somewhere along this anatomical pathway (Fig. 1A). This could involve changes at the level of the primary sensory response at peripheral sites in the lips and tentacles and/or changes in the processing of the CS in the CNS.

The anatomical organization of peripheral projections of the lip and tentacle nerves was studied by retrograde labeling. Backfilling the cut nerves toward the lips and tentacles revealed 
A

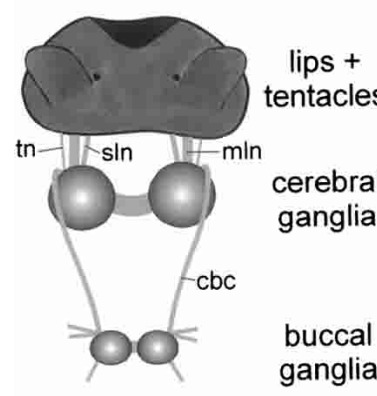

B

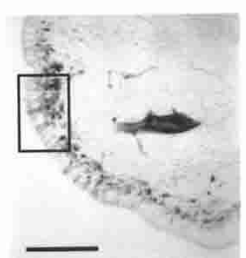

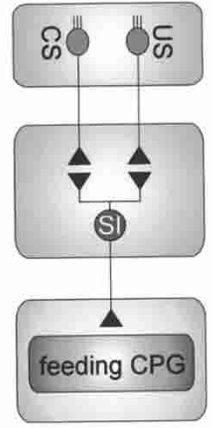

C

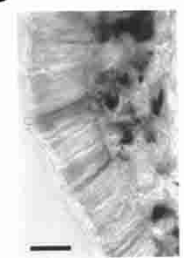

Figure 1. Organization of lip and tentacle sensory pathways to the CNS. ( $A$ ) Schematic diagram of anatomical organization of lip and tentacle sensory pathways. The lips and tentacles are connected to the cerebral ganglia via the median lip nerve $(\mathrm{mln})$, superior lip nerve $(\mathrm{s} / \mathrm{n})$, and tentacle nerve (tn). The cerebral-buccal connective (cbc) forms the only connection between the cerebral and buccal ganglia that contain the feeding CPG. Primary chemosensory cells in the lips and tentacles extend direct projections in the lip and tentacle nerves that synapse onto sensory integrating neurons (SI) in the cerebral ganglia (for simplicity, interactions are shown as direct connections). These neurons affect the feeding CPG in the buccal ganglia via the cerebral-buccal connective. $(B)$ Photomicrograph of putative primary chemosensory cells labeled by backfilling the median lip nerve in a lip section. The picture shows biocytin-labeled neuronal cell bodies arranged in a subepithelial layer. Scale bar, $100 \mu \mathrm{m}$. (C) Enlargement of area marked by square in $B$. Note the fine processes originating from the labeled subepithelial cell bodies that project through the lip epithelium. Scale bar, $20 \mu \mathrm{m}$.

a large number of sensory cells with cell bodies located in the subepithelial layer (Fig. 1B). Fine dendrites extend through the sensory epithelium, where they terminate externally in fine processes (Fig. 1C). These are typical of chemosensory neurons and are comparable to previously described chemoreceptors in Lymnaea (see Zaitseva and Bocharova 1981). These data suggest that primary chemosensory neurons in the lips and tentacles extend axons directly to the cerebral ganglia via the lip and tentacle nerves.

Extracellular recordings were made from the lip and tentacle nerves to determine experimentally whether conditioning results in peripheral changes in the chemosensory responses. Removing the central ganglia eliminated efferent activity from the CNS. This approach enabled us to record purely afferent responses to the application of the US and CS. In these multiunit recordings, population data on sensory responses were accumulated for each nerve. Initial experiments were carried out in naive animals to determine the basic responses to the US and CS. Subsequently sensory nerve recordings were made from conditioned and control animals.

In naive animals both the US and CS caused an increase in the activity recorded in both lip and tentacle nerves (Fig. 2A,B). These responses showed some variability between individual preparations. However, the averaged response patterns in all three nerves and for both stimuli were similar, consisting of an increase in spiking activity that declined rapidly when the stimu-

lus was removed (Fig. 2C,D). A statistical analysis revealed no significant difference in the average response of the three nerves to either the US or CS (two way ANOVA: $F_{(5,7)}=0.63, P=0.68$ ). Although the sensory nerve response patterns to the US and CS could not be discriminated on the basis of the overall changes in nerve activity, the nerves must carry different information in response to the two stimuli because the US activates strong feeding behavior in naive animals whereas the CS does not (Kemenes et al. 2002).

Next, to assess the possible contribution of overall changes in nerve activity to the conditioned response, we recorded sensory nerve responses to the US and CS in conditioned and control animals. Behavioral tests made prior to recording revealed marked differences in feeding responses to the CS between conditioned and control animals. Thus, following conditioning, feeding scores (measured as the difference in the number of feeding movements in the 2 min prior to CS application minus the number of feeding movements in the 2 min after the CS) to the CS were $17.1 \pm 3.9$ rasps $/ 2 \mathrm{~min}$ in conditioned animals and $0.7 \pm 1.1 \mathrm{rasps} / 2 \mathrm{~min}$ in control animals ( $t$ test, $P<0.01 ; \mathrm{n}=7$ ). This difference in behavioral response to the CS in conditioned and control animals was not, however, reflected in the sensory nerve responses to the CS. The time course and amplitude of the sensory nerve responses in the conditioned and control animals were not significantly different (Fig. 3A-C; median lip nerve: control $1.6 \pm 0.2 \mathrm{~Hz}$ versus conditioned $1.2 \pm 0.2 \mathrm{~Hz}$; superior lip nerve: control $1.9 \pm 0.2 \mathrm{~Hz}$ versus conditioned $1.1 \pm 0.3 \mathrm{~Hz}$; tentacle nerve: control $1.1 \pm 0.2 \mathrm{~Hz}$ versus conditioned $0.6 \pm 0.1 \mathrm{~Hz}$; $t$ tests: $P>0.05, \mathrm{n}=7$ for control and conditioned preparations). Furthermore, the overall CS responses in these two groups of animals were comparable to the lip and tentacle nerve responses recorded in naive animals (cf. Figs. 2 and 3). The marked difference in the behavioral responsiveness to the CS in conditioned and control animals therefore cannot be attributed to changes in the overall afferent activity pattern entering the CNS.

\section{Conditioning alters central representation of the CS}

Information from the lips and tentacles first enters the CNS via the cerebral ganglia, but it must reach the buccal ganglia in order to activate the feeding CPG (Fig. 1A). As the cbc is the only route from the cerebral to the buccal ganglia, this connective was used to monitor the effects of conditioning on information processing within the cerebral ganglia. So, we recorded activity traveling from the cerebral to the buccal ganglia in semi-intact preparations made from naive, conditioned, and control animals.

In naive preparations the US evokes a strong increase in activity traveling in the connective from the cerebral to the buccal ganglia (Fig. 4A,C). The time course of the spike frequency increase shows some variability between individual preparations, and this showed itself particularly in the duration of the response, which is longer on average in the combined data of Figure 4C compared with the individual trace of Figure 4A. In contrast to the US, the CS caused no significant change in the activity recorded in the cbc (mean change in cbc activity: $0.05 \pm 0.21 \mathrm{~Hz} ; t$ test, $P>0.05 ; \mathrm{n}=7$; Fig. 4B,C). Furthermore, the change in cbc frequency in response to the CS was also significantly different from the US response $(1.33 \pm 0.56 \mathrm{~Hz}, \mathrm{n}=5 ; t$ test, $P>0.05)$. The US responses were consistently present in the cbc despite the lack of response to the CS when both stimuli were applied in an alternating sequence to the same preparation. Increasing the concentration of the CS from $0.5 \mathrm{mM}$ to $2.7 \mathrm{mM}$ also failed to cause a change in the mean activity (data not shown). From this it is clear that in naive animals information related to the CS and US entering the cerebral ganglia is processed very differently. Naive snails perceive the CS, but the peripheral sensory information 
A

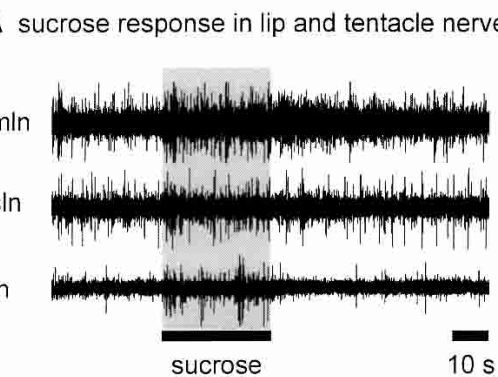

C

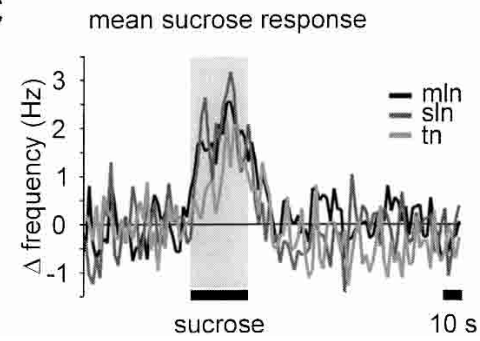

B

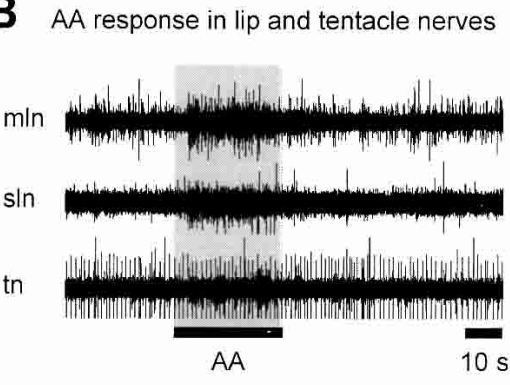

D

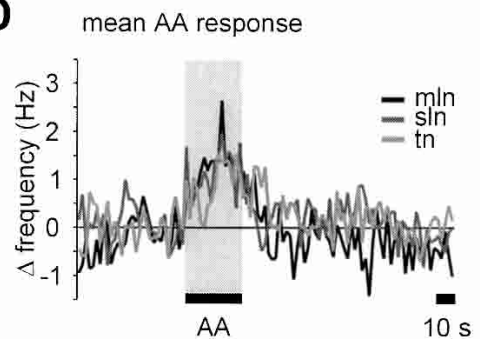

Figure 2. Sensory nerves in naive snails show similar responses to sucrose and AA application to the lips. (A) Simultaneous recordings from median lip nerve $(\mathrm{mln})$, superior lip nerve (sln), and tentacle nerve $(\mathrm{tn})$ showing responses of the three nerves to sucrose $(0.02 \mathrm{M}, 30 \mathrm{sec})$ applied to the lips. Note that the lip and tentacle nerves showed a considerable level of spontaneous activity that varied to some extent between individual preparations, including both tonic firing and spontaneous bursts of activity. The level of spontaneous activity did not influence the overall responses of the nerves to chemical stimulation of the lips. All nerves to the lips were cut to remove efferent influences from the CNS. (B) Equivalent record from another preparation showing responses of the same three nerves to AA $(0.54 \mathrm{mM}, 30 \mathrm{sec})$ applied to the lips. It should be noted that the two unidentified larger units that fire at an almost constant frequency throughout the record contributed only a small proportion to the overall nerve activity and that the main change in activity occurred in the small to medium sized units. $(C, D)$ Average time course of changes in nerve firing rates in median lip nerve, superior lip nerve and tentacle nerve in response to sucrose $(C, n=7$ preparations; error bars not shown for clarity) and $\mathrm{AA}(D, \mathrm{n}=7$ preparations; error bars not shown for clarity) application.

does not activate the cerebral-buccal pathway. Sensory information in the cerebral ganglia related to the US, on the other hand, does activate the cerebral-buccal pathway. This suggests that synaptic connectivity within the cerebral ganglia is important in the stimulus classification that determines whether the feeding CPG in the buccal ganglia is activated or not.

The dependence of the transmission of chemosensory information to the buccal ganglia on synaptic connectivity was demonstrated by bathing the CNS in nominally zero calcium saline (Fig. 5A). This treatment completely blocked the response to the US. The block was highly significant (Fig. 5B; one-way ANOVA for correlated samples: $P<0.01$, followed by Tukey HSD test: control versus zero calcium: $P<0.05$; zero calcium versus wash: $P<0.01, \mathrm{n}=6$ ). This finding is consistent with the anatomical organization of the primary afferent projections of the lip and tentacle nerves, which terminate in the cerebral ganglia (Fig. 1A, right).

These results suggest a systems-level model in which synaptic connectivity in the cerebral ganglia is altered by conditioning, allowing information related to the CS to reach the feeding CPG in conditioned animals. To examine this, we recorded the output of the cerebral ganglia in response to the $\mathrm{CS}$ after conditioning in control and conditioned animals (Fig. 6A,B). Successful conditioning was confirmed by behavioral testing. Difference feeding scores for the conditioned group were $9.0 \pm 2.9 \mathrm{rasps} / 2 \mathrm{~min}$ and for the control group $0.9 \pm 1.8 \mathrm{rasps} / 2 \mathrm{~min}$ ( $t$ test, $P<0.05$; $\mathrm{n}=9$ ). In marked contrast to the result obtained in naive animals, the CS application in conditioned animals caused a con- sistent increase in the activity of the cbc. This difference was specific to the CS response as both groups showed comparable average sucrose responses (Fig. 6C,D) that were also similar to the average sucrose responses observed in naive animals (Fig. 4C). The comparison between the average CS and the US responses in the conditioned group also shows the overall similarities in the time course of the two responses. A statistical comparison of the average changes in total cbc activity during the $30 \mathrm{sec}$ of stimulus application showed that the CS response in control animals $(0.0 \pm 0.7 \mathrm{~Hz}$, $\mathrm{n}=8$ ) was significantly different from the CS response in conditioned animals $(1.8 \pm 0.41 \mathrm{~Hz}, \mathrm{n}=6)$ as well as the US responses in control and conditioned animals $(2.1 \pm 0.5 \mathrm{~Hz}, \mathrm{n}=8$ and $3.1 \pm 0.6 \mathrm{~Hz}$, $\mathrm{n}=6$, respectively; ANOVA $F_{(3,24)}=7.68$, $P<0.01$, followed by post hoc Games/ Howell tests, $P<0.05)$. Furthermore, it revealed that the average CS response in conditioned animals and the US responses in control and conditioned animals were not significantly different (post hoc Games/ Howell tests, $P>0.05)$.

\section{Discussion}

The main aim of this study was to investigate the potential contribution of peripheral and central changes in the processing of chemosensory information leading to long-term memory formation. Our results show that appetitive conditioning does not significantly alter peripheral processing of the CS and US. Chemosensory information from the periphery is integrated and processed centrally first in the cerebral ganglia. In naive animals information about the US is transmitted onto the buccal ganglia, where it can activate the feeding CPG. Information about the CS in naive animals, however, is filtered in the cerebral ganglia and does not reach the buccal ganglia. Nonetheless, the central filtering/processing of the CS is plastic and can be altered by experience enabling the CS to access the feeding CPG in conditioned animals.

Our results do not rule out peripheral processing of chemosensory information in Lymnaea. In fact, although Lymnaea lacks peripheral sensory ganglia such as those present in terrestrial snails (Chase 2002), ultrastructural studies do show peripheral synapses between sensory neurons (Roubos and van der WalDivendal 1982). Their functional significance is unclear, but our results suggest that these synapses are unaffected by appetitive conditioning and do not contribute significantly to the conditioned response. We cannot completely rule out the possibility of peripheral synaptic plasticity, but this would be difficult to test experimentally. The recording of population responses by extracellular methods did not allow the identification of individual units, so conditioning-induced changes in a small number of sensory fibers could have been missed.

We propose the following model for appetitive conditioning that also takes into account the neuroanatomical organization of the sensory structures in Lymnaea. Chemosensory cells in the lips and tentacles extend primary afferent axons into the cerebral ganglia consistent with previous anatomical studies of chemosensory structures and pathways in Lymnaea (Zaitseva and Bo- 


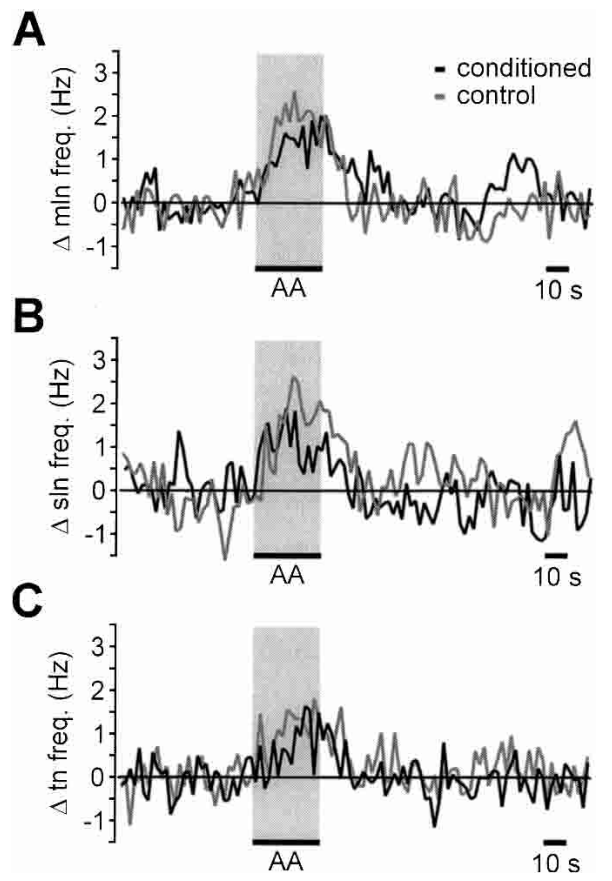

Figure 3. Sensory nerve responses to lip stimulation with $A A$ are not significantly different in preparations from control and conditioned snails. $(A, B, C)$ Average change in nerve activity in response to $\mathrm{AA}(0.54 \mathrm{mM}, 30$ sec) applied to the lips recorded in the median lip nerve $(A)$, superior lip nerve $(B)$, and tentacle nerve $(C)$ of control and conditioned snails $(n=7$ for each nerve; error bars not shown for clarity).

charova 1981; Roubos and van der Wal-Divendal 1982). We assume that there are subpopulations of chemosensory cells that differ in their responsiveness to various chemical stimuli. Thus, different chemical stimuli (e.g., the US and CS) will activate distinct sets of chemosensory cells, which synapse first onto central integrating interneurons in the cerebral ganglia. These integrating interneurons relay sensory information to other parts of the CNS and elicit behavioral responses appropriate to the chemical stimulus. In Lymnaea the activation of the subset of integrating neurons that respond to sucrose for example will lead to the initiation of the feeding behavior. In naive animals, however, the CS does not activate this pathway. This might be due to the lack or weakness of functional synapses between CS sensitive sensory neurons and sensory integrating neurons, resulting in a central filtering of information about the CS. During appetitive conditioning, paired activity in the CS and US pathways selectively strengthens connections between CS responsive chemosensory neurons and sensory integrating interneurons that can activate the feeding CPG. These synaptic changes could occur in the preand/or postsynaptic neurons.

An alternative interpretation of our results is that a different population of peripheral sensory neurons responds to the CS after conditioning. However, in order to explain the lack of overall increase in nerve activity in the sensory nerves after conditioning, a subpopulation of sensory cells that are responsive to the CS in naive animals would have to be inhibited from responding to the CS. This type of explanation for our results would require a complex mechanism (peripheral and/or central) that balances facilitation and inhibition of the sensory responses in different subpopulations of sensory neurons after conditioning, and we are not aware of any sensory system that has such features. The model based on central plasticity provides a simpler explanation of the results and can be tested experimentally in the future.
Our Lymnaea model is similar to a number of other systems, including terrestrial mollusks, insects, and mammals, that show changes in the central processing of chemosensory information following learning. In all these systems, chemosensory learning results in changes in the early stages of central processing, for example, the procerebral lobes in terrestrial mollusks (Kimura et al. 1998; Ermentrout et al. 2001), the antennal lobes in insects (see Daly et al. 2001; Menzel 2001; Sandoz et al. 2003), and the olfactory bulb in mammals (see Lledo and Gheusi, 2003; Yuan et al. 2003; Martin et al. 2004). Appetitive tactile conditioning in Lymnaea using mechanical stimulation of the lips as the CS also leads to an increase in the cbc CS response following conditioning (Staras et al. 1999). Despite differences between the conditioned cbc responses in tactile and chemical appetitive conditioning (e.g., time course, duration; most likely due to differences in the stimuli nature), it shows that tactile conditioning also involves changes in the early processing stage of sensory information.

In these systems, however, it is not known whether or not chemosensory learning also alters the peripheral sensory information entering the CNS. Studies of the cellular properties of olfactory receptors in salmon have shown that chemical imprint-

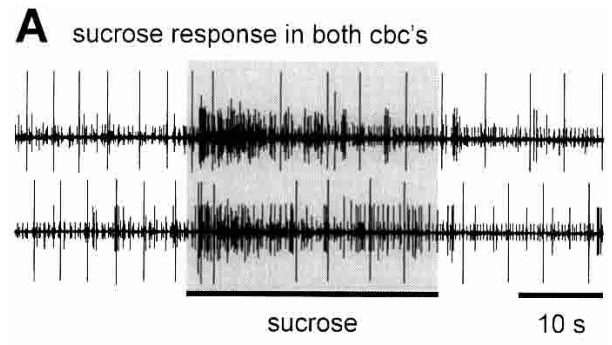

B lack of AA response in both cbc's

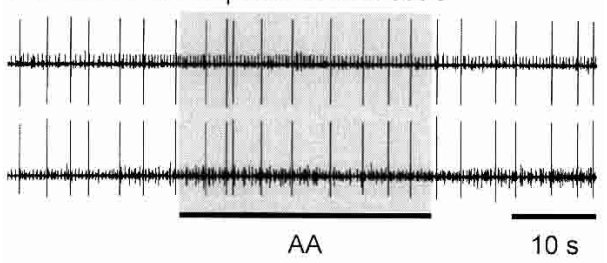

C

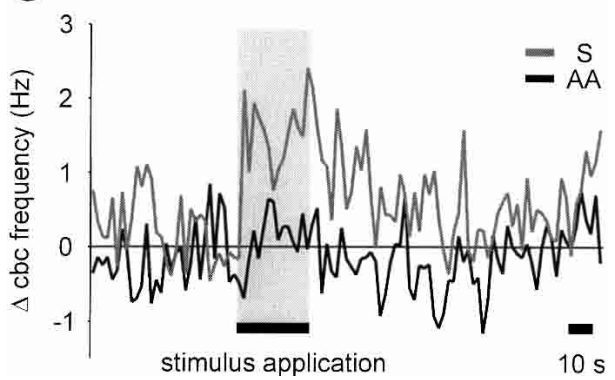

Figure 4. Sucrose, but not $A A$, application to the lips results in an increase of cerebral-buccal connective spike activity in preparations from naive snails. $(A)$ Simultaneous recording from both cut cerebral-buccal connectives showing the response of the two connectives to sucrose $(0.02 \mathrm{M}, 30 \mathrm{sec})$ applied to the lips. (B) Record from another preparation showing the lack of a cerebral-buccal connective response to AA (0.54 $\mathrm{mM}, 30 \mathrm{sec}$ ) applied to the lips in a naive preparation. (C) Time course of the average changes of spike activity in the cerebral-buccal connective in response to sucrose ( $n=5$ preparations) and $A A$ ( $n=7$ preparations) in naive snails (error bars not shown for clarity). The difference in the average frequency change during the $30 \mathrm{sec}$ of stimulus application was statistically significant. 
A
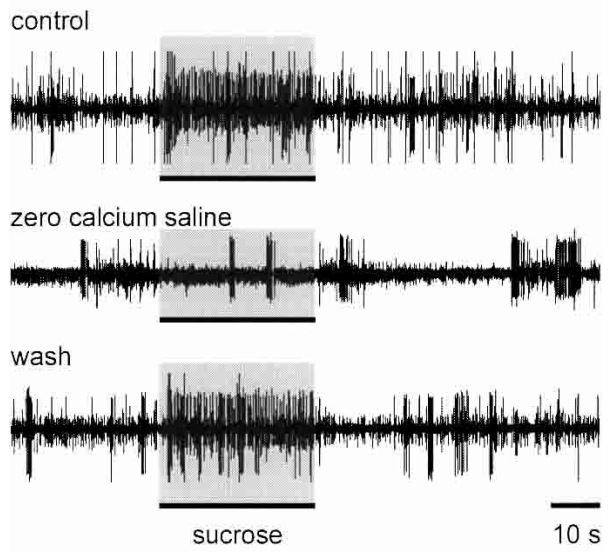

B

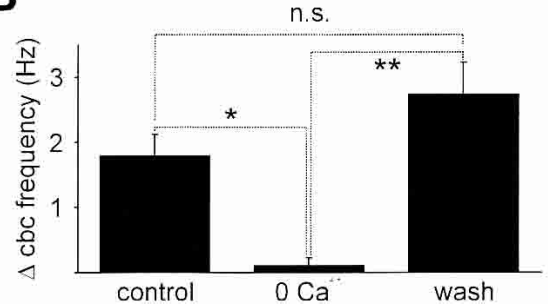

Figure 5. Response of cerebral-buccal connective to lip application of sucrose is blocked by zero calcium saline, suggesting that it is mediated by chemical synapses in the cerebral ganglia. $(A)$ Sample records from a cut cerebral-buccal connective showing responses to sucrose $(0.02 \mathrm{M}, 30$ $\mathrm{sec}$ ) applied to the lips in normal saline (top trace), in nominally zero calcium saline (middle trace), and after return to normal saline (bottom trace). Note the reduced levels of spontaneous activity and the lack of an obvious response to sucrose application in zero calcium saline. (B)The summary of average changes in cerebral-buccal connective activity during the period of sucrose application shows that zero calcium saline applied to the cerebral ganglia completely abolishes the sucrose response in the cerebral-buccal connective (one-way ANOVA for correlated samples, $P<0.01$ followed by post-hoc Tukey HSD tests, control versus zero calcium: $P<0.05$, zero calcium versus wash: $P<0.01 ; n=6$ preparations).

ing can lead to changes in the intrinsic properties of chemoreceptor cells (Nevitt et al. 1994). Similarly, conditioning of the light response in the marine snail Hermissenda results in changes of the cellular properties of photoreceptors, including an increase in excitability (Alkon et al. 1985). An increase in input resistance and excitability of mechanosensory neurons was also found after classical conditioning of the siphon withdrawal reflex in Aplysia (Antonov et al. 2001). Learning can therefore potentially affect primary sensory cells in a way that would lead to a change in their firing properties and could affect the response pattern of a sensory nerve. We directly address this question and demonstrate that in Lymnaea the firing patterns of the sensory nerves are unaffected by chemical conditioning, whereas firing patterns of a central connective are strongly affected, indicating a change in the central processing of chemosensory information.

Our model predicts the existence of integrating interneurons that process chemosensory information and activate stimuli-specific behaviors. In Lymnaea, various cerebral-buccal interneurons have been identified including the cerebral giant cell (CGC) and the cerebral ventral 1 (CV1) interneuron (McCrohan 1984; Benjamin and Elliott 1989; Yeoman et al. 1994a,b). Future work will concentrate on the identification of the predicted integrating interneurons and the characterization of their responsiveness to US and CS stimulation in naive and conditioned animals.

\section{Materials and Methods}

\section{Animals}

All experiments described here were carried out using adult (45-mo-old) pond snails, Lymnaea stagnalis. The animals were bred and raised in the Lymnaea breeding facilities at the University of Sussex, where they were kept at a temperature of $20^{\circ} \mathrm{C}$ in a 12-h light/12-h dark cycle and fed a diet of lettuce and fish food.

\section{One-trial appetitive conditioning of Lymnaea}

One-trial appetitive conditioning of Lymnaea was carried out by using a training procedure, based on previously described protocols (Kemenes et al. 2002). Two groups of animals were included in each experiment. The conditioned group received a paired presentation of the CS AA and US sucrose, whereas the control received a single presentation of the CS AA followed by a single presentation of the US sucrose $1 \mathrm{~h}$ later. Prior to training snails were starved for $4 \mathrm{~d}$.

Training protocol for unpaired control group: For training, individual snails were positioned in the center of a $15-\mathrm{cm}$ Petri dish filled with clean Cu-free water $(90 \mathrm{~mL})$ and left for $10 \mathrm{~min}$. At this point, the CS (5 mL $0.08 \%$ AA) was added to the dish, which was followed $20 \mathrm{sec}$ later by the addition of a neutral water stimulus $(5 \mathrm{~mL})$ so that the dish contained $100 \mathrm{~mL}$ solution with a final AA concentration of $0.27 \mathrm{mM}$. The animals were removed from these dishes $2 \mathrm{~min}$ after the addition of the CS and returned to a tank containing clean water. After 50 min individual animals in the unpaired group were again put in clean Petri dishes filled with water $(90 \mathrm{~mL})$ and left for $10 \mathrm{~min}$. At this point, a neutral water stimulus $(5 \mathrm{~mL})$ was added to each dish followed $20 \mathrm{sec}$ later by the US $(5 \mathrm{~mL} 0.4 \mathrm{M}$; final sucrose concentration in dish,0.02 M). Again, the animals were removed from the dishes 2 min after the addition of the first stimulus, that is, the neutral water stimulus, and transferred to a tank containing clean water to rinse off any chemical stimuli. After $10 \mathrm{~min}$ the animals were transferred to another tank containing clean water, where they remained until testing $24 \mathrm{~h}$ later.

Training protocol for paired group: All handling and timing intervals were essentially the same as for the unpaired group, whereas the order of stimuli applications was altered. Ten minutes after individual animals in the paired group were placed in Petri dishes containing clean water $(90 \mathrm{~mL})$, a first neutral water stimulus $(5 \mathrm{~mL})$ was applied followed by a second identical neutral water stimulus $20 \mathrm{sec}$ later. The animals were removed from the Petri dishes 2 min after the application of the first water stimulus and transferred to a tank with clean water. Fifty minutes later, individual snails were again placed in clean Petri dishes filled with water and left for $10 \mathrm{~min}$, when the CS (5 mL 0.08\% AA) was added to the dish. The CS was followed $20 \mathrm{sec}$ later by the application of the US ( $5 \mathrm{~mL} 0.4 \mathrm{M}$; final dish volume, $100 \mathrm{~mL}$; final AA and sucrose concentration in dish, $0.27 \mathrm{mM}$ and $0.02 \mathrm{M}$, respectively). The animals were transferred to a clean water tank 2 min after the addition of the CS, so that the animals were exposed to the CS alone for $20 \mathrm{sec}$ followed by a further 1-min 40 -sec period of overlapping exposure to the CS and US. After 10 min the animals were transferred to another tank containing clean water, where they remained until testing $24 \mathrm{~h}$ later.

The effect of the appetitive conditioning was tested "blind" $24 \mathrm{~h}$ after training. For testing, individual animals were placed for $10 \mathrm{~min}$ in $15-\mathrm{cm}$ Petri dishes filled with Cu-free water $(90 \mathrm{~mL})$. Each animal was then presented with a neutral water stimulus (5 $\mathrm{mL}$ water added to the dish), and the number of mouth openings followed by the protraction of the radula that constitute one feeding movement, or "rasp," was counted for a period of $2 \mathrm{~min}$. After the 2-min observation period, the CS (5 mL 0.08\% AA) was added to the dish, and the number of rasps was counted for another 2-min period. Afterward, animals were rinsed and returned to their home tanks. The difference feeding scores for each animal were calculated by subtracting the number of rasps 
A

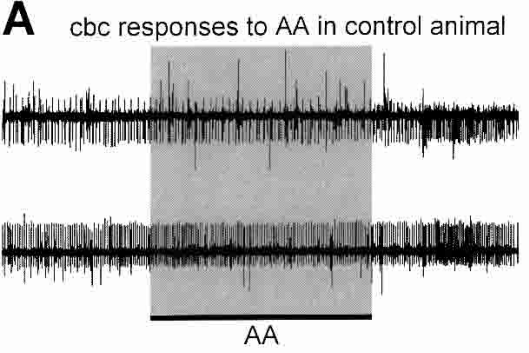

C

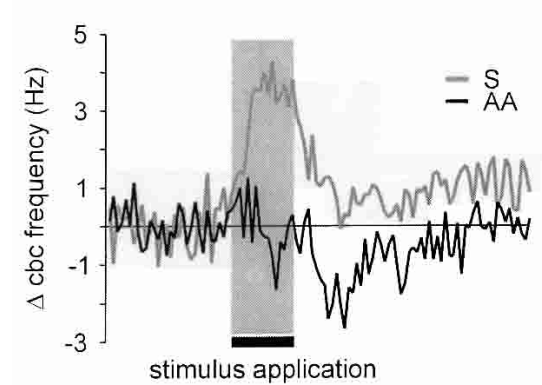

B

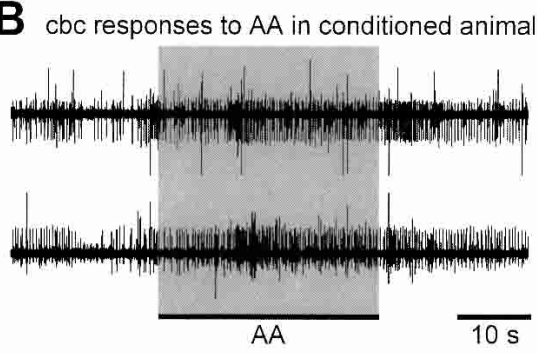

D

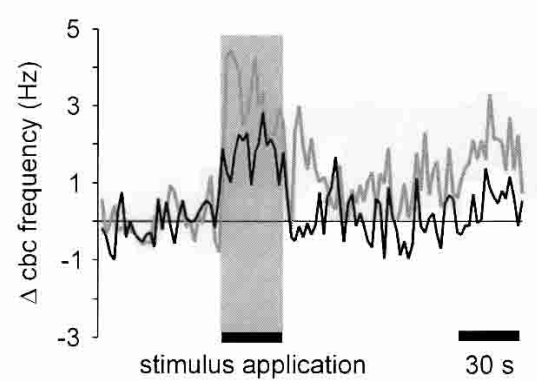

Figure 6. Appetitive conditioning enhances cerebral-buccal connective response to AA stimulation of the lips. $(A)$ Simultaneous recordings from the left and right cerebral-buccal connective in a preparation made from a control animal shows the lack of a response during application of AA. This is comparable to the situation in naive snails (see Fig. 4B). (B) Equivalent recordings from both cerebral-buccal connectives in a preparation made from a conditioned animal. Note the increase in overall activity in response to the application of $A A(0.54 \mathrm{mM}, 30 \mathrm{sec})$ to the lips. $(C, D)$ Time course of average changes in total cerebral-buccal connective spike activity in response to the CS and US in control and conditioned animals. The total change during the 30-sec period of CS application in control animals was significantly different from the CS response in conditioned animals and the US responses in both control and conditioned animals (ANOVA, $F_{(3,24)}=7.58$, $P<0.01$; post hoc Games/Howell tests, $P<0.05$ ). Further statistical analysis showed that the time course of the cerebral-buccal connective frequency changes during the US and CS application in conditioned animals was not significantly different (ANOVA for repeated measures, $P>0.05$ for within-subject effects [time], between-subject effects [group], and interaction effects [time $\times$ group]). An analysis of the time course of cerebral-buccal connective frequency changes during the US application in control and conditioned animals as well as in naive animals (Fig. 4C) also revealed no significant differences (ANOVA for repeated measures [Huynh-Feldt corrected], $P>0.05$ for within-subject effects [time], between-subjects effects [groups], and interaction effects [time $\times$ groups]).

an animal carried out after the neutral stimulus from the number of rasps an animal carried out in response to the CS. The difference feeding scores for all animals in the paired and unpaired group were averaged and compared using a standard $t$ test. Note also that the average number of rasps during the 2-min observation period after the neutral stimulus in the control and conditioned group for each experiment was not significantly different $(t$ tests, $P>0.05)$.

\section{Extracellular electrophysiological recording techniques}

The head of the snail plus the CNS was carefully dissected from the rest of the animal, making sure that the paired superior and medial lip nerves and tentacle nerves were left intact. All remaining peripheral nerves were cut. The semi-intact preparation was pinned in a Sylgard-lined recording chamber filled with snail saline (Straub et al. 2002). The outlet of a superfusion pipette was placed in front of the mouth region for constant superfusion of the preparation with fresh saline at a rate of $1-1.5 \mathrm{~mL} / \mathrm{min}$. Excess saline was removed from the opposite end of the recording chamber via a suction line. A set of electromagnetic valves that were controlled by the recording software enabled rapid switching between the saline stream and sucrose or AA solutions at various concentrations. When multiple chemical stimuli were tested on a single preparation, the order of application was randomized and interstimulus intervals were $10 \mathrm{~min}$.
Up to three independent extracellular suction electrodes were used to record spike activity from cut nerves. This set-up enabled the simultaneous recording of extracellular activity from the tentacle, superior, and medial lip nerves. In experiments that recorded peripheral activity from cut lip and tentacle nerves, not only the recorded nerves but all peripheral nerves were cut to remove the possibility of efferent feedback from the CNS via the remaining intact nerves. Two suction electrodes were used to record simultaneously activity in both cut cbc traveling from the cerebral ganglia to the buccal ganglia. In these experiments the tentacle, superior lip, and medial lip nerves were left intact.

Our experiments were aimed at the characterization of the early events that lead to the activation of feeding behavior in the intact animal and that are representations of the decision making processes in the animal's nervous system. Therefore, we decided to use shorter stimuli in these experiments than the test stimuli used in behavioral tests. Preliminary tests with various stimulus lengths had shown that 30-sec stimuli are sufficient to evoke consistent sensory responses in the peripheral sensory nerves.

Signals from the extracellular suction electrodes were differentially amplified by using Neurolog 104 amplifiers (Digitimer; gain, $10,000 \times$; AC coupled; time constant, $0.1 \mathrm{sec})$. The amplified signals were low pass $(500 \mathrm{~Hz})$ and notch $(50 \mathrm{~Hz})$ filtered by using Neurolog 125 filters (Digitimer) before they were digitized at a sampling rate of $2000 \mathrm{~Hz}$ using either an Axon TL-1 (Axon Instruments) or a CED 1401 (CED) interface and stored on a personal computer for subsequent analysis.

\section{Analysis of extracellular records}

Extracellular records were analyzed off-line by using Spike 2 (CED) software. The acquired raw data files were rectified, namely, all recorded values were replaced by absolute values, which resulted in records with only positive values. This procedure facilitated event detection as some units produced signals with larger positive amplitudes whereas signals from other units had larger negative amplitudes. A threshold was set manually at a level $\sim 20 \%$ larger than the maximal noise amplitude (peak-to-peak noise amplitude in most records was $<6$ $\mu \mathrm{V})$. All signal excursions that crossed the threshold and were spaced by $>10$ msec from a previous threshold crossing (to avoid multiple counting of multiphasic spikes) were automatically detected and counted as one event. The events were counted in 2-sec bins starting $60 \mathrm{sec}$ prior to and ending $150 \mathrm{sec}$ after the start of a stimulus application. For each record, the average activity for the 30 -sec period prior to a stimulus application was subtracted from each bin to give the net activity change in 2-sec bins induced by each stimulus.

For statistical comparisons between groups, the net changes for the $30 \mathrm{sec}$ of stimulus application were summed, giving the total net change during stimulus application, and the total net changes were compared by using standard statistical procedures.

\section{Test for synaptic interactions in cerebral ganglia}

Nominally zero calcium saline $\left(\mathrm{NaCl} 35 \mathrm{mM}, \mathrm{KCl} 1.6 \mathrm{mM}, \mathrm{MgCl}_{2}\right.$ $18 \mathrm{mM}$, HEPES $10 \mathrm{mM}$ ) was used to block central synaptic interactions and distinguish between the contribution of primary and 
secondary fibers to the sucrose responses observed in the cbc. Standard extracellular recording techniques as described above were used in these experiments. However, a Vaseline barrier build over the lip and tentacle nerves separated the peripheral lip tissue and the CNS, enabling the separate perfusion of the two structures. Initially, both compartments were perfused with standard snail saline and 30 -sec sucrose pulses at 10-min intervals were applied to the lips to establish the basic sucrose cbc response for each preparation. The CNS of preparations that showed consistent sucrose responses were then perfused with zero calcium saline, whereas the lips were perfused with standard saline and sucrose stimulation (30-sec pulses at 10 -min intervals) was continued. Perfusion of the lips with standard saline was important, as preliminary experiments had shown that perfusion of the lips with zero calcium saline blocked peripheral sensory responses, probably due to the effects of the lack of calcium on the chemosensory transduction process. Fifty minutes after the start of the zero calcium saline, perfusion of the CNS was switched back to standard saline. For statistical analysis, the three last sucrose responses prior to zero calcium application were averaged and compared with the average of the last three sucrose responses in zero calcium saline and the average of three sucrose responses starting $30 \mathrm{~min}$ after the return to standard saline.

\section{Anatomical studies of sensory nerve projections}

Biocytin was used to investigate the peripheral organization of the sensory neurons located in the skin of the lips and tentacles. Lip and tentacle nerves were severed close to the cerebral ganglia. The cut nerve of interest was sucked into a plastic pipette (pulled from fine bore polythene tubing, $0.5-\mathrm{mm}$ inner diameter, $1-\mathrm{mm}$ outer diameter) with a tip diameter slightly narrower than the nerve end. Saline solution in the pipette was replaced with a biocytin solution ( $2 \%$ in water), and the preparation was left overnight at $4^{\circ} \mathrm{C}$. The preparation was fixed in PFA (4\% in PBS) for $1 \mathrm{~h}$ at room temperature and dehydrated before it was cleared in histoclear, embedded in paraffin, and sectioned at 12 or 15 $\mu \mathrm{m}$. The sections were deparaffinized in histoclear, rehydrated, and rinsed in water. Biocytin was then visualized by using the $\mathrm{ABC}$ Vectastain kit.

\section{Acknowledgments}

The work was supported by a grant from the UK's Biotechnology and Biological Sciences Research Council. We would like to acknowledge the help of Drs. I. Kemenes and D. Fulton with the behavioral training experiments.

\section{References}

Alexander Jr., J., Audesirk, T.E., and Audesirk, G.J. 1984. One-trial reward learning in the snail Lymnaea stagnalis. J. Neurobiol. 15: $67-72$.

Alkon, D.L., Sakakibara, M., Forman, R., Harrigan, J., Lederhendler, I., and Farley, J. 1985. Reduction of two voltage-dependent $\mathrm{K}^{+}$currents mediates retention of a learned association. Behav. Neural. Biol. 44: $278-300$.

Antonov, I., Antonova, I., Kandel, E.R., and Hawkins, R.D. 2001. The contribution of activity-dependent synaptic plasticity to classical conditioning in Aplysia. J. Neurosci. 21: 6413-6422.

Benjamin, P.R. and Elliott, C.J.H. 1989. Snail feeding oscillator: The central pattern generator and its control by modulatory interneurons. In Neural and cellular oscillators (ed. J.W. Jacklet), pp. 173-214. Dekker, New York.

Benjamin, P.R., Staras, K., and Kemenes, G. 2000. A systems approach to the cellular analysis of associative learning in the pond snail Lymnaea. Learn. Mem. 7: 124-131.

Chase, R. 2002. Behavior and its neural control in gastropod molluscs. Oxford University Press, New York.

Christian, K.M. and Thompson, R.F. 2003. Neural substrates of eyeblink conditioning: Acquisition and retention. Learn. Mem. 10: 427-455.

Daly K.C., Chandra S., Durtschi M.L., and Smith B.H. 2001. The generalization of an olfactory-based conditioned response reveals unique but overlapping odor representations in the moth Manduca sexta. J. Exp. Biol. 204: 3085-3095.

Ermentrout, B., Wang, J.W., Flores, J., and Gelperin, A. 2001. Model for olfactory discrimination and learning in Limax procerebrum incorporating oscillatory dynamics and wave propagation. $J$. Neurophysiol. 85: 1444-1452.

Jones, N.G., Kemenes, I., Kemenes, G., and Benjamin, P.R. 2003. A persistent cellular change in a single modulatory neuron contributes to associative long-term memory. Curr. Biol. 13:1064-1069.

Kemenes, G., Staras, K., and Benjamin P.R. 1997. In vitro appetitive classical conditioning of the feeding response in the pond snail Lymnaea stagnalis. J. Neurophysiol. 78: 2351-2362.

. 2001. Multiple types of control by identified interneurons in a sensory-activated rhythmic motor pattern. J. Neurosci. 21: 2903-2911.

Kemenes, I., Kemenes, G., Andrew, R.J., Benjamin, P.R., and O'Shea, M. 2002. Critical time-window for NO-cGMP-dependent long-term memory formation after one-trial appetitive conditioning. $J$. Neurosci. 22: 1414-1425.

Kimura, T., Toda, S., Sekiguchi, T., and Kirino, Y. 1998. Behavioral modulation induced by food odor aversive conditioning and its influence on the olfactory responses of an oscillatory brain network in the slug Limax marginatus. Learn. Mem. 4: 365-375.

Lledo, P.M. and Gheusi, G. 2003. Olfactory processing in a changing brain. Neuroreport 14: 1655-1663.

Martin, C., Gervais, R., Hugues, E., Messaoudi, B., and Ravel, N. 2004. Learning modulation of odor-induced oscillatory responses in the rat olfactory bulb: A correlate of odor recognition? J. Neurosci. 24: 389-397.

McCrohan, C.R. 1984. Initiation of feeding motor output by an identified interneurone in the snail Lymnaea stagnalis. J. Exp. Biol. 113: 351-366.

Menzel, R. 2001. Searching for the memory trace in a mini-brain, the honeybee. Learn. Mem. 8: 53-62.

Mozzachiodi, R., Lechner, H.A., Baxter, D.A., and Byrne, J.H. 2003. In vitro analog of classical conditioning of feeding behavior in Aplysia. Learn. Mem. 10: 478-494.

Nakamura, H., Ito, I., Kojima, S., Fujito, Y., Suzuki, H., and Ito, E. 1999. Histological characterization of lip and tentacle nerves in Lymnaea stagnalis. Neurosci. Res. 33: 127-136.

Nevitt, G.A., Dittman, A.H., Quinn, T.P., and Moody Jr., W.J. 1994 Evidence for a peripheral olfactory memory in imprinted salmon. Proc. Natl. Acad. Sci. 91: 4288-4292.

Rosenzweig, M.R. 1996. Aspects of the search for neural mechanisms of memory. Annu. Rev. Psychol. 47: 1-32.

Roubos, E.W. and van der Wal-Divendal, R.M. 1982. Sensory input to growth stimulating neuroendocrine cells of Lymnaea stagnalis. Cell Tissue Res. 227: 371-386.

Sandoz, J.C., Galizia, C.G., and Menzel, R. 2003. Side-specific olfactory conditioning leads to more specific odor representation between sides but not within sides in the honeybee antennal lobes. Neuroscience 120: $1137-1148$.

Staras, K., Kemenes, G., and Benjamin, P.R. 1999. Cellular traces of behavioral classical conditioning can be recorded at several specific sites in a simple nervous system. J. Neurosci. 19: 347-357.

Straub, V.A., Staras, K., Kemenes, G., and Benjamin, P.R. 2002. Endogenous and network properties of Lymnaea feeding central pattern generator interneurons. J. Neurophysiol. 88: 1569-1583.

Yeoman, M.S., Kemenes, G., Benjamin, P.R., and Elliott, C.J. 1994a. Modulatory role for the serotonergic cerebral giant cells in the feeding system of the snail, Lymnaea, II: Photoinactivation. J. Neurophysiol. 72: 1372-1382.

Yeoman, M.S., Vehovszky, A., Kemenes, G., Elliott, C.J., and Benjamin, P.R. 1994b. Modulatory role for the serotonergic cerebral giant cells in the feeding system of the snail, Lymnaea, I: Fine wire recording in the intact animal and pharmacology. J. Neurophysiol. 72: 1357-1371.

Yuan, Q., Harley, C.W., Darby-King, A., Neve, R.L., and McLean, J.H. 2003. Early odor preference learning in the rat: Bidirectional effects of cAMP response element-binding protein (CREB) and mutant CREB support a causal role for phosphorylated CREB. J. Neurosci. 23: $4760-4765$.

Zaitseva, O.V. and Bocharova, L.S. 1981. Sensory cells in the head skin of pond snails. Cell Tissue Res. 220: 797-807.

Received March 12, 2004; accepted in revised form July 21, 2004. 


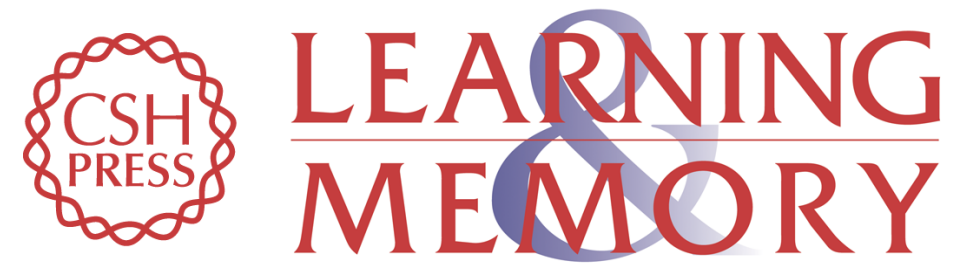

\section{Central localization of plasticity involved in appetitive conditioning in Lymnaea}

Volko A. Straub, Benjamin J. Styles, Julie S. Ireland, et al.

Learn. Mem. 2004, 11:

Access the most recent version at doi:10.1101/lm.77004

References This article cites 28 articles, 14 of which can be accessed free at:

http://learnmem.cshlp.org/content/11/6/787.full.html\#ref-list-1

License

Email Alerting Receive free email alerts when new articles cite this article - sign up in the box at the Service top right corner of the article or click here. 